\title{
Methoden zur Bestimmung von Wirkungsquerschnitten für Spinaustausch und Depolarisation indirekt optisch gepumpter Atome
}

\author{
A. Assfalg, J. Fricke, J. HaAs und E. Lüscher \\ Physik-Department der Technischen Universität München \\ (Z. Naturforsch. 26 a, 1571-1577 [1971] ; eingegangen am 2. Juni 1971)
}

\begin{abstract}
Two methods are described to derive cross sections for spin exchange and spin depolarization of an indirectly pumped alkali vapor with zero nuclear spin. Either pulsed or sinusoidally modulated radiofrequency can be used to destroy the polarization of the indirectly pumped (secondary) system, while the intensity of the transmitted pumping light is observed. Cross sections follow from the indirectly produced depolarization of the primary system i. e. from the change in pumping light transmission or from the phase angle between the rf modulation and the intensity oscillations of the transmitted light. The quality of the two methods is tested by deriving cross sections for spin exchange between $\mathrm{Cs}$ and $\mathrm{Rb}$ and for depolarization of $\mathrm{Rb}$ in Argon buffer gas.
\end{abstract}

\section{Einleitung}

Zur Bestimmung der Wirkungsquerschnitte der verschiedenen Relaxationsprozesse beim optischen Pumpen (Spinaustausch zwischen zwei Alkaliatomen, Depolarisation durch Stöße mit der Wand des Absorptionsgefäßes und durch Stoßprozesse zwischen einem Alkaliatom und einem Puffergasatom) muß der zeitliche Verlauf der Depolarisation, d. h. die Relaxationskurve, gemessen werden. Dazu wurden die von Franzen ${ }^{1}$ und Bouchiat und Grossetête ${ }^{2}$ angegebenen Verfahren verwendet.

Nach Franzen wird der Dampf bis zur Sättigung optisch gepumpt; dann schaltet man das Pumplicht kurzzeitig ab und läßt den Dampf im Dunkeln relaxieren. Die Intensität des transmittierten Pumplichts zum Zeitpunkt des Wiedereinschaltens ist ein $\mathrm{Maß}$ für den momentanen Polarisationsgrad des Systems. Wird nun die „Dunkelzeit" variiert, so kann man die Relaxationskurve punktweise aufnehmen. Bouchiat und Grossetête verfolgen die Depolarisation des Dampfes während der Dunkelzeit direkt mit Hilfe eines Detektionslichts, das so intensitätsschwach ist, daß es den Relaxationsverlauf nicht verändert. Im Prinzip genügt dann ein einmaliges Abschalten des Pumplichtes, um die Relaxationskurve vollständig aufzuzeichnen. Will man den Wirkungsquerschnitt für Depolarisation eines orientierten Atoms beim Stoß mit einem diamagnetischen Puffergasatom oder der Wand des Gefäßes bestimmen, so muß bei beiden Verfahren der Dampf „direkt“, d. h. durch Einstrahlung von zirkularpolarisiertem Resonanz-

Sonderdruckanforderungen an Prof. Dr. E. Lüscher, Physik-Department der Technischen Universität München, D-8046 Garching bei München. licht, optisch gepumpt werden. Dies bereitet im Fall der Alkaliatome keine Schwierigkeiten, da hierfür leistungsfähige Lichtquellen zur Verfügung stehen. Zum Nachweis der Polarisation des Dampfes durch Beobachtung des transmittierten Lichtes ist es notwendig, daß das eingestrahlte Resonanzlicht nicht alle Feinstrukturkomponenten im natürlichen Intensitätsverhältnis enthält. Diese Bedingung läßt sich bei den Alkalimetallen $\mathrm{Cs}$ und $\mathrm{Rb}$ bei Verwendung von Interferenzlinienfiltern leicht erfüllen. Oft läßt sich jedoch ohne Verwendung eines Monochromators oder sehr schmalbandiger Filter das natürliche Intensitätsverhältnis der Komponenten nicht genügend stark ändern. Vielfach sind auch die verfügbaren Pumplichtquellen so intensitätsschwach, daß es damit nicht gelingt, den Dampf merklich zu pumpen. In diesem Fall versucht man den Dampf „indirekt“, d. h. durch Spinaustausch mit einem zweiten Metalldampf (meist einem Alkalimetall), zu polarisieren, dessen Polarisation seinerseits direkt mit Resonanzlicht erzeugt und detektiert wird.

In der vorliegenden Arbeit werden zwei Methoden angegeben, um die Relaxationsrate eines indirekt gepumpten Dampfes infolge von Stößen mit Puffergasatomen und der Wand sowie den Spinaustauschwirkungsquerschnitt zu bestimmen.

\section{Ratengleichungen}

Die Meßverfahren basieren auf einem Modell für Alkaliatome $\left(S=\frac{1}{2}\right)$, bei dem die Kernspins beider Atomsorten A und B Null sind, d. h. es wird an-

1 W. Franzen, Phys. Rev. 115, 850 [1959].

2 M. A. Bouchiat u. F. Grossetête, J. Physique 27, 353 [1966]. 
genommen, daß die Optischen-Pump-Signale nur von der Polarisation der Elektronenhülle der Atome abhängen.

GIBBS $^{3}$ hat den Spinaustausch zwischen zwei realen Alkaliatomen $\left(S=\frac{1}{2}, I>0\right)$ untersucht und die Ergebnisse mit denen verglichen, die man erhält, wenn man den Kernspin vernachlässigt [ $(I=0)$-Modell]. Sie stimmen nur dann überein, wenn der Grundzustand bevorzugt nach dem von FRANZ ${ }^{4}$ angegebenen electron-randomization-Modell relaxiert.

Die Atomsorte A wird durch rechtszirkular polarisiertes $\mathrm{D}_{1}$-A-Licht optisch gepumpt. Durch Spinaustausch werden dann auch die B-Atome polarisiert. Der absorbierende A-Dampf soll optisch dünn sein; dann ist die Intensität $I_{0}$ des Pumplichts innerhalb der gesamten Absorptionszelle konstant. Die Zelle enthalte außerdem einige Torr eines diamagnetischen Puffergases und befinde sich in einem schwachen Magnetfeld $H_{0}$, das parallel zur Einstrahlungsrichtung des Pumplichtes verlaufe. Senkrecht zu $H_{0}$ werde ein magnetisches Wechselfeld $H_{1}(\omega)$ eingestrahlt, das mit der Zeeman-Resonanzfrequenz $\omega$ der B-Atome schwingt.

Die Differentialgleichungen für die zeitlichen Änderungen der Polarisation $a(t)$ bzw. $b(t)$ des Abzw. B-Ensembles ergeben sich dann folgendermaßen:

Es sei $a_{+}$bzw. $b_{+}$die Zahl der A- bzw. B-Atome pro $\mathrm{cm}^{3}$ mit $m_{\mathrm{s}}=+\frac{1}{2}$; entsprechend seien $a_{-}$und $b_{-}$ definiert. $N_{\mathrm{A}}$ bzw. $N_{\mathrm{B}}$ sei die Gesamtzahl der A- bzw. B-Atome pro $\mathrm{cm}^{3} \quad\left(N_{\mathrm{A}}=a_{+}+a_{-}, N_{\mathrm{B}}=b_{+}+b_{-}\right)$. Für die durch

$$
a=\frac{a_{+}-a_{-}}{a_{+}+a_{-}}=\frac{a_{+}-a_{-}}{N_{\mathrm{A}}} \quad \text { und } \quad b=\frac{b_{+}-b_{-}}{N_{\mathrm{B}}}
$$

definierten Polarisationen erhält man die beiden gekoppelten Differentialgleichungen (vgl. ${ }^{5}$ )

$$
\begin{aligned}
& \dot{a}=k I_{0}(1-a)-2 r_{\mathrm{A}} a-v_{\mathrm{r}} Q N_{\mathrm{B}}(a-b), \\
& \dot{b}=-2 r_{\mathrm{B}} b+v_{\mathrm{r}} Q N_{\mathrm{A}}(a-b)-2 R b .
\end{aligned}
$$

Dabei ist $k$ eine Konstante, welche die Absorptionswahrscheinlichkeit für das Pumplicht enthält und den Grad des Mixing im angeregten Zustand von A beschreibt (vgl. $\left.{ }^{6}\right) . r_{\mathrm{A}}$ und $r_{\mathrm{B}}$ sind die Relaxationsraten der A- bzw. B-Atome infolge von Stößen mit Puffergasatomen und der Wand der Absorptionszelle. Stöße zwischen gleichartigen Atomen brauchen

3 H. Gibbs, Phys. Rev. 139, A 1374 [1965].

${ }^{4}$ F. A. Franz, Phys. Rev. 141, 105 [1966].

5 S. M. Jarrett, Phys. Rev. 133, A 111 [1964]. nicht berücksichtigt zu werden, da sie in der $(I=0)$ Näherung keine Änderung der resultierenden Polarisation eines Ensembles bewirken. $Q$ ist der über alle Relativgeschwindigkeiten gemittelte Wirkungsquerschnitt für Spinaustausch bei A-B-Stößen und $v_{\mathrm{r}}$ eine gemittelte Relativgeschwindigkeit zwischen Aund B-Atomen. $R$ ist die Relaxationsrate der BAtome infolge Einstrahlung der Resonanzfrequenz. $R$ ist proportional zu $H_{1}^{2} f\left(\omega-\omega_{0}\right)$, wobei $f\left(\omega-\omega_{0}\right)$ eine „Formfunktion“ ist, die von der Differenz der eingestrahlten Frequenz $\omega$ und der exakten Resonanzfrequenz $\omega_{0}$ abhängt. Die Gestalt dieses Ausdrucks ist jedoch für alle Betrachtungen unwichtig.
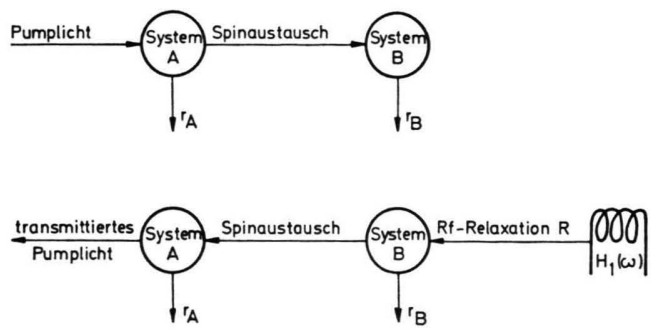

Abb. 1. (a) Blockbild des Pumpvorgangs, (b) Blockbild des Detektionsvorgangs.

Abbildung 1 zeigt zur Veranschaulichung der Verhältnisse Blockbilder des Pump- und Detektionsvorganges.

\section{Die Pulsmethode}

Bei diesem Meßverfahren wird der B-Dampf durch rechteckförmige Resonanzfrequenzpulse (typische Dauer: 0,5 sec) depolarisiert. Abbildung 2 zeigt den zeitlichen Verlauf von $R(t)$ und den Polarisationen $b(t)$ und $a(t)$. Vor dem Einschalten der Resonanzfrequenz liegen die Polarisationen $a_{0}$ und $b_{0}$ vor. Nach dem Einschalten fallen $b(t)$ und a $(t)$ ab. $a(t)$ kann durch Beobachtung des transmittierten Pumplichts direkt verfolgt werden (Abb. 2).

Im folgenden interessiert nur das Zeitintervall $\tau_{1} \leqq t \leqq \tau_{2}$, in dem $R(t)=r=$ const ist. $\tau_{1}$ wird als Zeitnullpunkt angenommen $\left(\tau_{1}=0\right)$.

Aus den Gln. (1) und (2) ergibt sich als Lösung:

$$
a(t)=k_{1} e^{-\Theta_{1} t}+k_{2} e^{-\Theta_{2} t}+a_{\min } .
$$

Dabei ist

$$
\Theta_{1,2}=\frac{\beta+\delta}{2} \pm \sqrt{\left(\frac{\beta+\delta}{2}\right)-\Omega_{0}^{2}}, \quad \Theta_{1}>\Theta_{2}
$$

6 J. Fricke, J. HaAs, E. Lüscher u. F. A. Franz, Phys. Rev. 163, 45 [1967]. 


$$
a_{\min }=\alpha \delta / \Omega_{0}^{2}
$$

mit

$$
\begin{aligned}
\alpha & =k I_{0}, \\
\beta & =k I_{0}+2 r_{\mathrm{A}}+v_{\mathrm{r}} Q N_{\mathrm{B}}, \\
\gamma & =v_{\mathrm{r}} Q N_{\mathrm{A}}, \\
\delta & =2 r_{\mathrm{B}}+v_{\mathrm{r}} Q N_{\mathrm{A}}+2 r, \\
\varepsilon & =v_{\mathrm{r}} Q N_{\mathrm{B}}, \\
\Omega_{0}^{2} & =\beta \delta-\gamma \varepsilon, \\
\eta & =\beta+\delta .
\end{aligned}
$$

$k_{1}$ und $k_{2}$ hängen von den Anfangsbedingungen des Systems ab und können im Vorzeichen wechseln; dieses Problem wird im Anhang betrachtet. Bei den Testmessungen an dem $\mathrm{Cs}-\mathrm{Rb}-\mathrm{Ar}-\mathrm{System}$ waren $k_{1}$ und $k_{2}$ immer positiv.

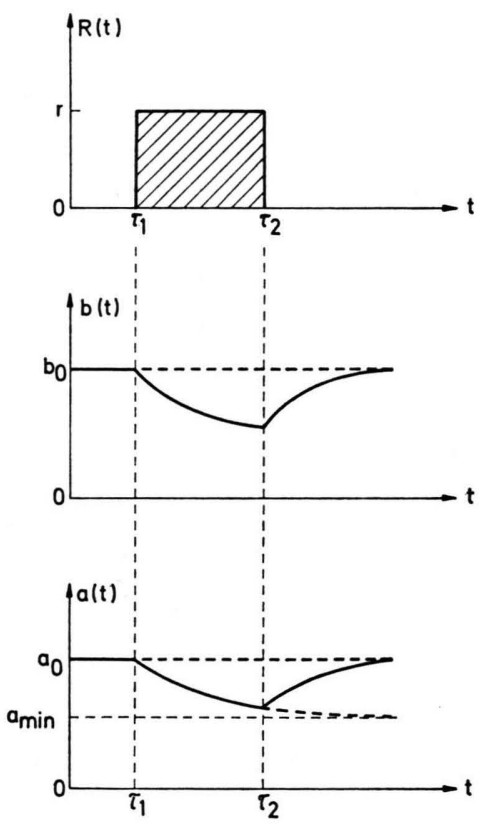

Abb. 2. Zeitlicher Verlauf von $R(t)$ und der Polarisationen $b(t)$ und $a(t)$ bei der Pulsmethode.

Die Kurve $a(t)$ [Gl. (3) ] kann bis auf einen Verstärkungsfaktor $V$ direkt mittels Photomultiplier und Oszillograph oder Vielkanal-Analysator aufgenommen werden. Die Information über die interessierenden Größen steckt in den beiden Zeitkonstanten $\Theta_{1}$ und $\Theta_{2}$, den Konstanten $k_{1}$ und $k_{2}$ und in $a_{\min }$. Nun ist es nicht einfach, Absolutwerte einer Polarisation zu bestimmen; $a_{\min }$ entfällt also als Informationsquelle. $k_{1}$ und $k_{2}$ sind ebenfalls unbrauchbar, da die Anfangsbedingungen des Systems sehr schwer zu erfassen sind. Alle Daten müssen daher den beiden Zeitkonstanten entnommen werden.

Ist die Pulslänge so groß, daß das System die Gleichgewichtspolarisation $a_{\min }$ annimmt, so kann man diesen Wert als neue Null-Linie wählen und dann die Größen $\Theta_{1}$ und $\Theta_{2}$ zeichnerisch bestimmen.

Wie die Definitionen (4) und (5) zeigen, ergibt sich durch Addition von $\Theta_{1}$ und $\Theta_{2}$ die Größe

$$
\eta=\beta+\delta=\Theta_{1}+\Theta_{2}
$$

und durch Multiplikation

$$
\Omega_{0}^{2}=\beta \delta-\gamma \varepsilon=\Theta_{1} \Theta_{2} .
$$

Verändert man die Höhe des Rechteckpulses, dann geht $r$ über in einen anderen Wert $r^{+}=r+\Delta r$ und $\delta \rightarrow \delta^{+}=\delta+2 \Delta r$. Mit diesem veränderten Puls wird nun eine neue $a(t)$-Kurve aufgenommen und aus ihr die Größen

und

$$
\eta^{+}=\beta+\delta^{+}=\eta+2 \Delta r
$$

$$
\Omega_{0}^{+2}=\beta \delta^{+}-\gamma \varepsilon=\Omega_{0}^{2}+2 \beta \Delta r
$$

bestimmt.

Aus diesen beiden Gleichungen folgt

$$
\beta=\left(\Omega_{0}^{+2}-\Omega_{0}^{2}\right) /\left(\eta^{+}-\eta\right)=k I_{0}+2 r_{\mathrm{A}}+v_{\mathrm{r}} Q N_{\mathrm{B}}
$$

und damit

$$
\delta=\eta-\beta \equiv \eta-\left(\Omega_{0}^{+2}-\Omega_{0}^{2}\right) /\left(\eta^{+}-\eta\right) .
$$

Unter Verwendung dieser beiden Ergebnisse ergibt sich dann aus Gl. (7) :

$\gamma \varepsilon=\frac{\Omega_{0}^{+2}-\Omega_{0}^{2}}{\eta^{+}-\eta}\left(\eta-\frac{\Omega_{0}^{+2}-\Omega_{0}^{2}}{\eta^{+}-\eta}\right)-\Omega_{0}^{2}=v_{\mathrm{r}}^{2} Q^{2} N_{\mathrm{A}} N_{\mathrm{B}}$

und daraus $Q=\sqrt{\gamma \varepsilon} / v_{\mathrm{r}} \sqrt{N_{\mathrm{A}} N_{\mathrm{B}}}$.

Variiert man die Amplitude des Resonanz-Rechteckpulses - und damit $r$-, so ergibt sich $\delta$ als Funktion der Rf-Amplitude. Eine Extrapolation dieser Kurve nach $r \rightarrow 0$ gibt $2 r_{\mathrm{B}}+v_{\mathrm{r}} Q N_{\mathrm{A}}$, woraus mit (12) die Relaxationsrate $r_{\mathrm{B}}$ folgt. Den Messungen kann man außerdem auch die Abhängigkeit der Relaxationsrate $r$ von der Rf-Amplitude entnehmen [aus $\Delta r=\frac{1}{2}\left(\delta^{+}-\delta\right)$ und dem Wert von $\delta_{r=0}=2 r_{\mathrm{B}}$ $\left.+v_{\mathrm{r}} Q N_{\mathrm{A}}\right]$. Um den Wirkungsquerschnitt für Spinaustausch $Q$ zu bestimmen, ist also im Prinzip nur die Kurve $a(t)$ für zwei verschiedene Pulshöhen aufzunehmen. Dies kann, z. B. mit Hilfe eines Signalanalysators, in wenigen Minuten geschehen. Während dieser kurzen Zeit können die Bedingungen (Inten- 
sität der Pumplichtquelle, Dampfdrücke,...) sehr gut konstant gehalten werden. Zur Bestimmung von $r_{\mathrm{B}}$ sind Messungen bei verschiedenen Pulshöhen erforderlich (Extrapolation).

Für kleine Rf-Amplituden läßt sich diese Methode nicht mehr anwenden, da das Signal-Rausch-Verhältnis zu klein wird. Mit der im nächsten Abschnitt geschilderten „Phasenmethode“" lassen sich jedoch auch Messungen für kleine Rf-Amplituden durchführen.

\section{Die Phasenmethode}

Bei diesem Verfahren wird die Resonanzfrequenz und damit die Relaxationsrate $R$ des B-Grundzustandes sinusförmig mit der Frequenz $\Omega$ moduliert.

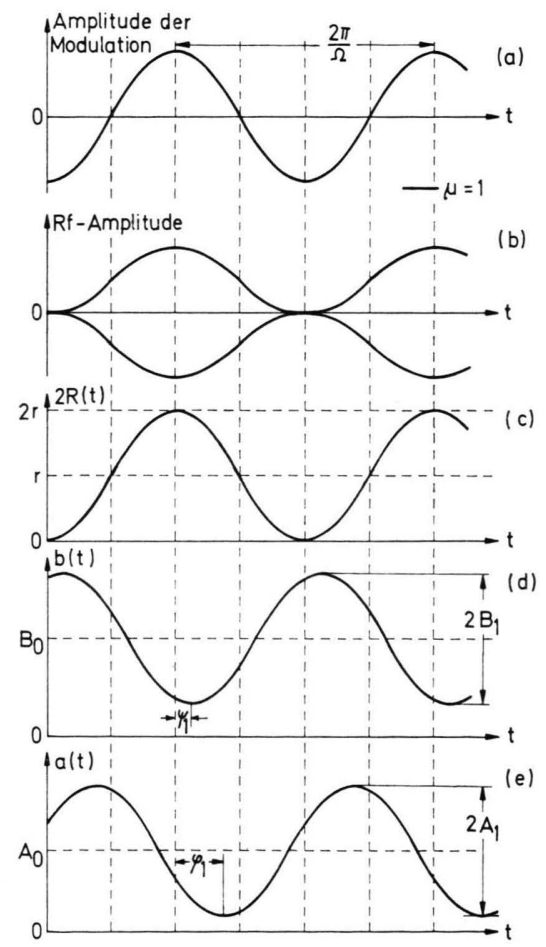

Abb. 3. Zeitlicher Verlauf der Rf-Amplitude, der Relaxationsrate $R(t)$ und der Polarisation $b(t)$ und $a(t)$ bei der Phasenmethode.

Abbildung 3 zeigt die Rf-Amplitude, die Relaxationsrate $R$ und die Polarisationen $b$ und $a$ als Funktion der Zeit $t$. In komplexer Darstellung wird der Term $2 R$ aus Gl. (2) jetzt ersetzt durch

$$
2 R=r\left(1+\mu e^{i \Omega t}\right) \text {. }
$$

$r$ ist gegeben durch die Amplitude der unmodulierten Rf, $\mu$ ist der Modulationsgrad $(0 \leqq \mu \leqq 1)$,
$\Omega$ die Modulationsfrequenz. Um möglichst große Signale zu erhalten, wird man $\mu=1$ wählen (vgl. Abb. 3) ; für die Meßmethode selbst ist es jedoch völlig unerheblich, wie groß der Modulationsgrad ist.

Die Differentialgleichungen (1) und (2) gehen unter Verwendung der Abkürzung (5) über in

$$
\begin{aligned}
& \dot{a}=\alpha+\beta a+\gamma b, \\
& \dot{b}=-\delta^{\prime} \beta+\varepsilon a-\mu r e^{i \Omega t} b
\end{aligned}
$$

mit

$$
\delta^{\prime}=2 r_{\mathrm{B}}+v_{\mathrm{r}} Q N_{\mathrm{A}}+r .
$$

Zur Lösung dieses Dgl.-Systems werden für $a(t)$ und $b(t)$ Fourier-Reihen angesetzt:

$a(t)=A_{0}+A_{1} \cdot e^{i\left(\Omega t+\varphi_{1}\right)}+A_{2} \cdot e^{i\left(2 \Omega t+\varphi_{2}\right)}+\ldots$,

$b(t)=B_{0}+B_{1} \cdot e^{i\left(\Omega t+\psi_{1}\right)}+B_{2} \cdot e^{i\left(2 \Omega t+\psi_{2}\right)}+\ldots$.

Die Winkel $\varphi_{1}, \varphi_{2}, \ldots$ und $\psi_{1}, \psi_{2}, \ldots$ geben die Phasenverschiebungen der Polarisationen gegenüber der Phase der Modulation an (vgl. Abb. 3). Durch Beobachtung des transmittierten Pumplichts sind $\varphi_{1}, \varphi_{2}, \ldots$ und Relativwerte für $A_{0}, A_{1}, A_{2}, \ldots$ meßbar.

Mit diesen Ansätzen erhält man aus den Gln. (13) und (14) die Beziehungen

$$
\operatorname{ctg} \varphi_{1}=\left(\Omega^{2}-\Omega_{0}^{\prime 2}\right) / \eta^{\prime} \Omega
$$

und

$$
A_{1} / \cos \varphi_{1}=\mu r \gamma B_{0} /\left(\Omega^{2}-\Omega_{0}{ }^{\prime 2}\right) .
$$

Dabei wurde $\eta^{\prime}=\beta+\delta^{\prime}$ und $\Omega_{0}{ }^{\prime 2}=\beta \delta^{\prime}-\gamma \varepsilon$ gesetzt. $\varphi_{1}$ nimmt Werte zwischen 0 und $-2 \pi$ an.

Für $\Omega=\Omega_{0}^{\prime}$ ist $\operatorname{ctg} \varphi_{1}=0$, d. h. $\varphi_{1}=-90^{\circ}$. Mißt man daher für einige Modulationsfrequenzen - bei fester Rf-Amplitude - die Phasenverschiebung $\varphi_{1}$, so kann aus dem $\left(-90^{\circ}\right)$-Durchgang von $\varphi_{1}$ die Größe $\Omega_{0}{ }^{\prime 2}$ bestimmt werden. Bei bekannten $\Omega_{0}^{\prime 2}$ läßt sich dann mit einem $\left(\Omega, \varphi_{1}\right)$-Paar aus Gl. (17) $\eta^{\prime}=\beta+\delta^{\prime}$ berechnen. Die Situation ist nun dieselbe wie beim Pulsverfahren. Man verändert $r \rightarrow r^{*}=r+\Delta r$ und erhält die Größen

und

$$
\begin{aligned}
\Omega_{0}{ }^{* 2} & =\beta \delta^{*}-\gamma \varepsilon \\
\eta^{*} & =\beta+\delta^{*} .
\end{aligned}
$$

Aus diesen 4 Größen lassen sich dann mit den Gln. (10), (11) und (12) wieder $\beta, \delta$ und $\gamma \varepsilon$ bestimmen.

Die Messung der $\left(-90^{\circ}\right)$-Durchgänge ist unnötig, wenn man neben $\varphi_{1}$ auch Relativwerte (!) von $A_{1}$ mißt. Der Modulationsfrequenz $\Omega_{1}$ seien $A_{11}$ und $\varphi_{11}$, der Frequenz $\Omega_{2}$ seien $A_{12}$ und $\varphi_{12}$ zuge- 
ordnet. Gleichung (18) zeigt dann:

$$
\frac{A_{11} / \cos \varphi_{11}}{A_{12} / \cos \varphi_{12}}=\frac{\Omega_{2}{ }^{2}-\Omega_{0}{ }^{2}}{\Omega_{1}{ }^{2}-\Omega_{0}{ }^{2}} .
$$

Hieraus wird $\Omega_{0}^{\prime 2}$ berechnet; Gl. (17) liefert dann das zugehörige $\eta^{\prime}$.

\section{Experimente}

Mit den beschriebenen Methoden wurden Testmessungen an einem $\mathrm{Cs}-\mathrm{Rb}-\mathrm{Ar}$-System durchgeführt. Der Cs-Dampf wurde direkt mit Cs $-\mathrm{D}_{1}-\sigma^{+}$-Licht gepumpt, die Rb-Atome wurden durch Spinaustausch polarisiert (d. h. $\mathrm{Cs}=\mathrm{A}$-Atom, $\mathrm{Rb}=\mathrm{B}-$ Atom). Bei allen Messungen wurde natürliches $\mathrm{Rb}$ verwendet; dieses besteht zu $72,15 \%$ aus $\mathrm{Rb}^{85}$ ( $I=\frac{5}{2},\left|g_{\mathrm{F}}\right|=\frac{1}{3}$ im Grundzustand) und zu $27,85 \%$ aus $\operatorname{Rb}^{87}\left(I=\frac{3}{2},\left|g_{\mathrm{F}}\right|=\frac{1}{2}\right.$ im Grundzustand). Der Versuchsaufbau entsprach einer üblichen Apparatur für optisches Pumpen (vgl. z. B. ${ }^{7}$ ). Der Argondruck betrug etwa 8,4 Torr; die Zelle wurde auf einer Temperatur von $40^{\circ} \mathrm{C}$ gehalten.

\section{a) Pulsmethode}

Die Messungen wurden mit der in Abb. 4 schematisch dargestellten Anordnung durchgeführt. Die Rf-Pulse

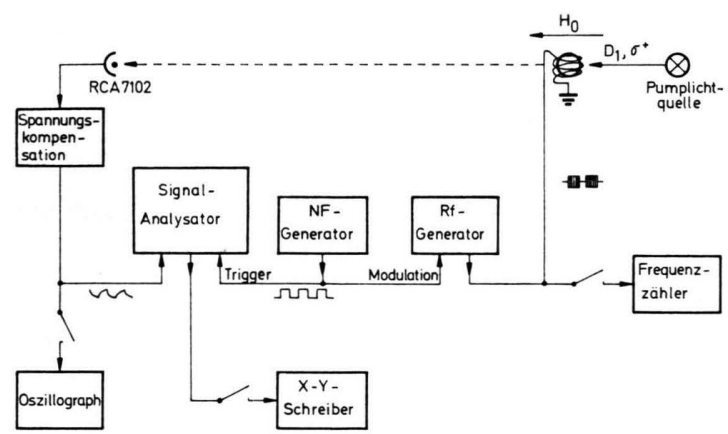

Abb. 4. Schematischer Aufbau der Apparatur bei der Pulsmethode.

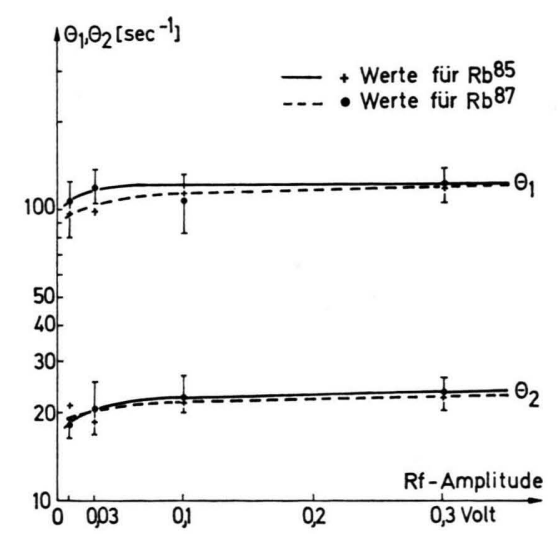

Abb. 5. Die Zeitkonstanten $\Theta_{1}$ und $\Theta_{2}$ als Funktionen der Rf-Amplitude für Cs-Rb ${ }^{85}$ und $\mathrm{Cs}-\mathrm{Rb}^{87}$. haben eine Pulsdauer von 0,5 sec und eine Wiederholfrequenz von $1 \mathrm{~Hz}$. Die moduliernde Rechteckspannung wird auch zum Triggern des Signalanalysators verwendet. Gleichspannungsanteile des Multipliersignals werden mit einer variablen Spannungsquelle wegkompensiert. Für jede Rf-Amplitude wurden 64 Durchläufe aufgenommen, und die vom Signalanalysator gemittelten Kurven dann mit dem $X$ - $Y$-Schreiber gezeichnet. Aus der Asymptote für $t \approx 0,5 \mathrm{sec}$ wurde die „NullLinie“ $\left(a_{\min }=0\right)$ bestimmt.

Abbildung 5 zeigt den Verlauf von $\Theta_{1}$ und $\Theta_{2}$ als Funktion der Pulshöhe für $\mathrm{Rb}^{85}$ und $\mathrm{Rb}^{87}$.

\section{b) Phasenmethode}

Die verwendete Schaltung ist in Abb. 6 dargestellt. Zur Modulation der Resonanzfrequenz wird der interne Sinusgenerator des Lock-in-Verstärkers (Typ PAR Modell HR-8) verwendet. Wenn der Rf-Generator gleichstromgekoppelt ist, entsteht keine Phasendifferenz zwischen der modulierten Rf, d. h. der Rf-induzierten Relaxationsrate $R(t)$, und dem Modulationssignal. Das Modulationssignal kann daher als Referenzsignal verwendet werden, von welchem aus der gesuchte Phasenwinkel $\varphi_{1}$ gerechnet wird.

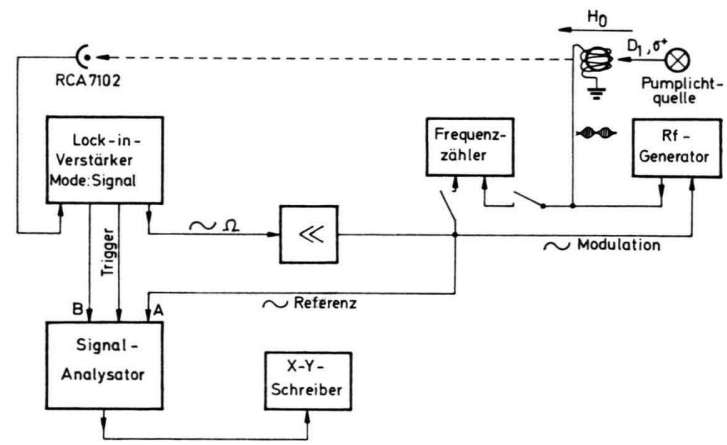

Abb. 6. Schematischer Aufbau der Apparatur zur Messung nach der Phasenmethode.

Der Lock-in-Verstärker wird nur als Schmalbandverstärker - also nicht phasenempfindlich - betrieben. Bei dieser Einstellung erscheint an seinem Ausgang ein Sinussignal der Frequenz $\Omega \quad(\Omega=$ Modulationsfrequenz), dessen Amplitude proportional ist zur Amplitude der $\Omega$-Fourier-Komponente des Multipliersignals. Der Signalanalysator wird mit einer Rechteckspannung (synchron zur modulierenden Spannung, die dem Calibration-output des Lock-in-Verstärkers entnommen wird), getriggert.

$\varphi_{1}$ wird durch Abmessen der Verschiebung der Nulldurchgänge bestimmt. Bei der Auswertung muß noch berücksichtigt werden, daß der Lock-in-Verstärker selbst eine Phasenverschiebung erzeugt, die in einem Vorversuch ermittelt wird.

Die Messungen wurden nur an dem häufigeren $\mathbf{R b}^{85}$ durchgeführt. Abbildung 7 zeigt die Phasenverschie-

7 W. W. Holloway, E. Lüscher u. R. Novick, Phys. Rev. 126, 2109 [1962]. 
bung $\varphi_{1}$, Abb. 8 die Fourier-Amplitude $A_{1}$ als Funktion der Modulationsfrequenz.

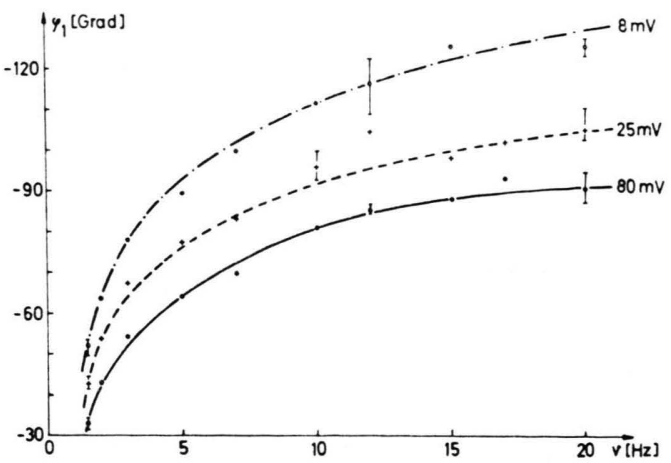

Abb. 7. $\varphi_{1}$ als Funktion der Modulationsfrequenz für Cs-Rb ${ }^{85}$. Parameter ist die Rf-Amplitude.

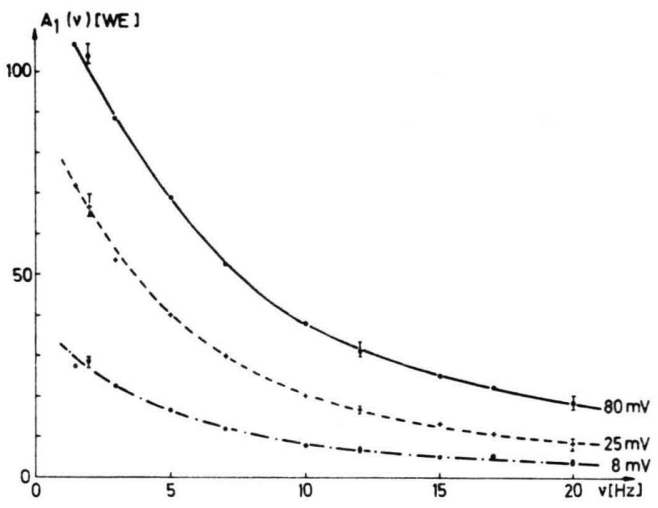

Abb. 8. Relativwerte von $A_{1}$ als Funktion der Modulationsfrequenz. Parameter ist die Rf-Amplitude.

\section{Ergebnisse und Diskussion}

Mit der Pulsmethode ergaben sich für die Wirkungsquerschnitte für Spinaustausch die Werte

und

$$
Q\left(\mathrm{Cs}, \mathrm{Rb}^{85}\right)=(1,45 \pm 0,30) \cdot 10^{-14} \mathrm{~cm}^{2}
$$

$$
Q\left(\mathrm{Cs}, \mathrm{Rb}^{87}\right)=(1,6 \pm 0,3) \cdot 10^{-14} \mathrm{~cm}^{2} .
$$

Die Relaxationsrate $r_{\mathrm{Rb}}$ wurde abgeschätzt zu

$$
r_{\mathrm{Rb}} \approx 16 \mathrm{sec}^{-1} \text {, }
$$

woraus sich wegen $1 / T_{1}=2 r_{\mathrm{Rb}}$ eine Depolarisationszeit von etwa 31 msec ergibt. Die Relaxationsrate setzt sich zusammen aus einem Beitrag der Rb-Ar. Stöße $r_{\mathrm{Rb}, \Lambda \mathrm{r}}$ und einem Anteil $r_{\mathrm{Rb}, \mathrm{w}}$ aus Rb - WandStößen:

$$
r_{\mathrm{Rb}}=r_{\mathrm{Rb}, \mathrm{Ar}}+r_{\mathrm{Rb}, \mathrm{W}} \cdot
$$

8 R. A. Bernheim, J. Chem. Phys. 36, 135 [1962].

9 M. Arditi u. T. R. Carver, Phys. Rev. 136, A 643 [1964].
Bei einer runden Zelle vom Radius $\varrho$ gilt nach BERNHEIM $^{8}$ näherungsweise:

$$
r_{\mathrm{Rb}, \mathrm{W}}=\frac{\pi^{2}}{\varrho^{2}} D_{0} \frac{760}{p} .
$$

Dabei ist $D_{0}$ die Diffusionskonstante bei einem Puffergasdruck von 760 Torr und $p$ der tatsächliche Puffergasdruck in Torr.

Die Diffusionskonstante $D_{0}$ hat nach ${ }^{9}$ ungefähr den Wert $D_{0}=0,2 \mathrm{~cm}^{2} / \mathrm{sec}$; damit läßt sich $r_{\mathrm{Rb}, \mathrm{W}}$ und $r_{\mathrm{Rb}, \text { Ar }}$ berechnen $(\varrho=3,9 \mathrm{~cm})$. Aus

$$
r_{\mathrm{Rb}, \mathrm{Ar}}=v_{\mathrm{rel}} Q(\mathrm{Rb}, \mathrm{Ar}) N_{\mathrm{Ar}}
$$

ergibt sich dann:

$$
Q(\mathrm{Rb}, \mathrm{Ar}) \approx 2 \cdot 10^{-22} \mathrm{~cm}^{2} .
$$

Die Phasenmethode liefert

$$
Q\left(\mathrm{Cs}, \mathrm{Rb}^{85}\right)=(1,3 \pm 0,3) \cdot 10^{-14} \mathrm{~cm}^{2} .
$$

Bei zusätzlicher Verwendung von $A_{1}$-Werten erhält man als Wirkungsquerschnitt für Spinaustausch den Wert

$$
Q\left(\mathrm{Cs}, \mathrm{Rb}^{85}\right)=(1,1 \pm 0,2) \cdot 10^{-14} \mathrm{~cm}^{2} .
$$

Für den Depolarisationsquerschnitt erhält man

$$
Q(\mathrm{Rb}, \mathrm{Ar}) \approx 6 \cdot 10^{-22} \mathrm{~cm}^{2} \text {. }
$$

Als Wirkungsquerschnitt für Spinaustausch zwischen Cs- und Rb-Atomen haben Bouchiat und GrosseTÊTE ${ }^{2}$ die Werte

$$
Q\left(\mathrm{Cs}, \mathrm{Rb}^{85}\right)=Q\left(\mathrm{Cs}, \mathrm{Rb}^{87}\right) \approx 2,4 \cdot 10^{-14} \mathrm{~cm}^{2}
$$

gemessen. GibBs und Hull ${ }^{10}$ geben

$$
Q\left(\mathrm{Cs}, \mathrm{Rb}^{87}\right)=(2,3 \pm 0,2) \cdot 10^{-14} \mathrm{~cm}^{2}
$$

an. Nach ${ }^{9}$ ist $Q\left(\mathrm{Rb}^{87}-\mathrm{Ar}\right) \approx 9 \cdot 10^{-22} \mathrm{~cm}^{2}$, nach ${ }^{1}$ hat $Q(\mathrm{Rb}-\mathrm{Ar})$ den Wert $3,7 \cdot 10^{-22} \mathrm{~cm}^{2}$ und nach ${ }^{11}$ ist $Q(\mathrm{Rb}-\mathrm{Ar}) \approx 1,1 \cdot 10^{-22} \mathrm{~cm}^{2}$. Die für die Wirkungsquerschnitte für Spinaustausch zwischen $\mathrm{Cs}$ - und $\mathrm{Rb}^{85}$-, bzw. $\mathrm{Rb}^{87}$-Atomen gemessenen Werte sind nur um etwa $35 \%$ kleiner als die von Bouchiat und Grossetête ${ }^{2}$ bzw. Gibbs und Hull ${ }^{10}$ angegeben. Damit zeigt sich, daß die zur Größenordnungsbestimmung gedachte Methode brauchbare Resultate liefert. Eine Ursache für die Abweichung dürfte in den vereinfachenden Annahmen des verwendeten Modells liegen. Gibbs zeigte, daß die mit Hilfe eines $(I=0)$-Modells erhaltenen Ergebnisse für die Wirkungsquerschnitte für Spin-

10 H. M. Gibbs u. R. J. Hull, Phys. Rev. 153, 132 [1967].

11 F. A. Franz, Phys. Rev. 139, A 603 [1965]. 
austausch bis um den Faktor 6,8 von den richtigen Werten abweichen können. Weitere Abweichungen können aus Ungenauigkeiten der Dampfdichten ent. stehen.

Ziel der vorliegenden Arbeit war es, Meßmethoden zu entwickeln, die ein Studium der Relaxation und des Spinaustausches von indirekt gepumpten Atomen beispielsweise mit inneren unaufgefüllten Schalen (Europium ${ }^{12,13}$, Mangan ${ }^{14}$ ) erlauben; denn selbst die Größenordnung der Wirkungsquerschnitte für Spinaustausch und Depolarisation durch Puffergasstöße ist bei solchen Atomen noch nicht bekannt. An einer Verfeinerung der Meßmethodik durch Nachweis von Polarisation und Ausrichtung eines durch Spinaustausch gepumpten Eu- oder Mn-Dampfes wird gearbeitet (vgl. 13, 14).

\section{Anhang}

Im folgenden sollen die Vorzeichen der Konstanten $k_{1}$ und $k_{2}$ aus Gl. (3) diskutiert werden.

Vor dem Einschalten des Pulses hat $b$ den Wert $b_{0}=\alpha \varepsilon /\left.\Omega_{0}{ }^{2}\right|_{r=0}$ und $a$ den Wert $a_{0}=\alpha \delta /\left.\Omega_{0}{ }^{2}\right|_{r=0}$. Nun werde die Rf zur Zeit $t=0$ eingeschaltet. Die Amplitude - und damit die Relaxationsrate $R$ erreicht jedoch nicht sofort ihren vollen Wert $r$, sondern erst nach einer gewissen Anstiegszeit $\tau_{\mathrm{A}}$. $\mathrm{Zu}$ dieser Zeit hat die Polarisation der B-Atome den Wert $b\left(\tau_{\mathrm{A}}\right)=(1-f) b_{0}$, mit $0<f<2$, da das resultierende magnetische Moment sich unter dem Einfluß des ansteigenden $H_{1}$-Feldes aus der $H_{0}$-Richtung herausgedreht hat. Die Polarisation der A-Atome ist in guter Näherung unverändert geblieben $\left[a\left(\tau_{\mathrm{A}}\right)\right.$ $\left.=a_{0}\right]$, da in der Zeit $\tau_{\mathrm{A}} \approx 10^{-5}$ sec noch kein merklicher Spinaustausch stattgefunden hat. Da alle Rechnungen sich auf die volle Pulshöhe und damit maximale Relaxationsrate $r$ beziehen, die jedoch erst zur Zeit $t=\tau_{\mathrm{A}}$ vorliegt, müssen als Anfangsbedingungen

12 R. Tilgner, J. Fricke u. J. HaAs, Helv. Phys. Acta 42, 740 [1969].

13 R. Tilgner, J. Fricke u. J. HaAs, wird veröffentlicht. die Werte $a\left(\tau_{\mathrm{A}}\right)=a_{0}$ und $b\left(\tau_{\mathrm{A}}\right)=(1-f) b_{0}$ verwendet werden. Geht man damit in die Gln. (1), (2) und (3) ein, so ergeben sich für $k_{1}$ und $k_{2}$ die Beziehungen

$$
\begin{aligned}
& k_{1}=\frac{\gamma b_{0}}{\Theta_{1}\left(\Theta_{1}-\Theta_{2}\right)}\left(f \Theta_{1}-2 r\right), \\
& k_{2}=\frac{\gamma b_{0}}{\Theta_{2}\left(\Theta_{1}-\Theta_{2}\right)}\left(2 r-f \Theta_{2}\right) .
\end{aligned}
$$

Ist $f=0$, d. h. steigt der Puls sehr schnell auf seinen vollen Wert an, so ist $k_{1}<0 . k_{1}$ wird jedoch positiv, wenn bei gegebener maximaler Pulshöhe, d. h. bei gegebenem $r$, die Ungleichung $f>2 r / \Theta_{1}$ gilt [Gln. (4) und (5) zeigen, daß $2 r<\Theta_{1}$ ist].

Hat sich während des Anstiegs des $H_{1}$-Feldes auf seinen vollen Wert das magnetische Moment aus der $H_{0}$-Richtung herausgedreht, so wird $f$ größer als Null.

Der Drehwinkel ergibt sich aus der Beziehung

$$
\hbar \omega=g \mu_{\mathrm{B}} H_{1 \text { eff }} \quad \text { zu } \quad \alpha=\frac{g \mu_{\mathrm{B}} H_{1} \text { eff }}{\hbar} \tau_{\mathrm{A}},
$$

wobei $H_{1 \text { eff }}$ ein gemitteltes Feld bedeutet, das auf das magnetische Moment dieselbe Wirkung hat wie das während der Anstiegszeit $\tau_{\mathrm{A}}$ anwachsende $H_{1}$ Feld $\left(H_{1 \text { eff }}=H_{1 \max } / \sqrt{3}\right)$; für die Relaxationszeit $\tau_{\mathrm{R}}$ des Systems gelte $\tau_{\mathrm{R}} \gg \tau_{\mathrm{A}}$. Im allgemeinen kann $f$ den maximalen Wert 2 annehmen. Gilt $2>f \geqq 1$, so wird $k_{2}$ bei positivem $k_{1}$ negativ. Bei einer Anstiegszeit von $\tau_{\mathrm{A}}=10^{-5} \mathrm{sec}$ liegt der Drehwinkel für $g=1$ in der Größenordnung von 10 bis $20^{\circ}$, für Kernspins ist er um den Faktor 1000 kleiner. Für Hüllenspins ist daher in der Regel $k_{1}$ positiv, für Kernspins negativ, was in der Tat beobachtet wurde ${ }^{15}$. Negative $k_{2}$ wurden nicht beobachtet.

Den Herren R. Tilgner und B.-H. Meyer danken wir für viele Diskussionen und Hinweise. Dem Bundesministerium für Bildung und Wissenschaft sind wir für die Gewährung von Sachmitteln zu Dank verpflichtet.

14 A. Assfalg, J. Fricke, J. HaAs, R. Tilgner u. E. Lüscher, wird veröffentlicht.

15 B.-H. MeYer, Diplomarbeit, Technische Universität München 1970. 